\title{
Acerca de una consideración naturalizada de la filosofía de la historia de Immanuel Kant: Epigénesis e historia universal
}

\author{
On a naturalized way of understanding Immanuel Kant's \\ philosophy of history: Epigenesis and universal history
}

Natalia Lerussi

natalialerussi@gmail.com

(Universidad de Buenos Aires, Buenos Aires, Argentina)

\begin{abstract}
Resumen: En el trabajo mostramos que la matriz conceptual utilizada por I. Kant en su concepción de la historia universal en su texto Idea de una historia universal en sentido cosmopolita es la misma mediante la cual el filósofo da cuenta de la producción de variaciones de los seres organizados en la historia natural, la teoría de la epigénesis (según las secciones $\$ \S 80$ y 81 de la Crítica de la facultad de juzgar). Defendemos así una concepción naturalizada de la filosofía de la historia kantiana.
\end{abstract}

Palabras claves: Kant; historia universal; disposiciones naturales; epigenesis.

\begin{abstract}
In the paper I show that universal history's conceptual matrix that I. Kant uses in his Idea of an Universal History from a Cosmopolitan Point of View is the same by which the philosopher explains the production of variations in the organic beings in natural history, the epigenesis theory (according to the sections $\$ \S 80$ y 81 of the Critique of the Power of Judgment). Consequently I defend a naturalized conception of Kant's philosophy of history.
\end{abstract}

Keywords: Kant; universal history; natural dispositions; epigenesist.

DOI: http://dx.doi.org/10.11606/issn.2318-9800.v20i1p93-105

\section{Introducción}

Se ha señalado recientemente que el concepto de "formación" (Bildung) implicado en la escuela de la epigenesis fue central en la gestación de la perspectiva que el siglo XVIII tuvo de la "formación cultural". ${ }^{1}$ En el presente trabajo indicaremos en qué sentido esta tesis es correcta a la luz de uno de los textos centrales en los que Kant aborda su concepción de la historia universal o historia de la cultura, esto es, en Idea de

1 Véase: WIEGEL, S. An der Schwelle von Kultur und Natur. Epigenetik und Evolutionstheorie. En: GEHARDT, V.; NIDA-RUMELIN (ed.). Evolution in Natur und Kultur. Berlin: De Gruyter, 2010, p. 118. El autor no menciona a Kant. 
una historia universal en sentido cosmopolita (Idea). ${ }^{2}$ Así mostraremos el sentido en el que la historia natural kantiana inscripta en el interior de la escuela de la epigénesis (o, como también el filósofo la denomina, el "preformismo genérico") define una matriz conceptual que permite comprender también la dinámica histórica o cultural. ${ }^{3}$ Específicamente, probaremos que Kant hace uso en su comprensión de la historia universal de los conceptos explicativos centrales que desarrolla para la comprensión de la producción de las variaciones o modificaciones en el interior de las especies naturales, e.d. la teoría de la epigénesis o de preformismo genérico. De allí que debemos mostrar que la dinámica de la historia universal, según Kant, se comprende a partir de los conceptos de "disposiciones naturales", "factores externos" propulsores de las mismas y de "fuerza formadora", los tres conceptos centrales de la epigénesis en la versión kantiana. En este sentido, entonces, estamos justificados a hablar de una consideración naturalizada de la filosofía de la historia kantiana.

En primer lugar (1), nos ocuparemos de mostrar qué habría entendido Kant por "epigénesis". Entonces mostraremos que son tres los elementos que supone esta escuela para explicar el origen de las variaciones de las especies naturales organizadas o seres orgánicos, en la versión del filósofo, esto es, una fuerza formadora, ciertas disposiciones originales y ciertas circunstancias externas. En la sección (2) presentaremos el rol explicativo fundamental que juegan las "disposiciones naturales" (para el uso de la razón) y las "circunstancias externas" en un texto central dedicado a la historia universal, esto es, en Idea. Luego, en la sección (3) haremos una breve

2 Aunque no tendremos aquí espacio para probarlo, este esquema también es el que Kant utiliza en los textos Presunto comienzo de la historia, la sección $\$ 83$ de la Crítica de la facultad de juzgar (CFJ), Sobre el dicho: esto puede ser cierto en la teoría pero no en la práctica y en El conflicto de las facultades. Otro modelo, aunque no podemos discutirlo aquí, es el utilizado en: Hacia la paz perpetua.

3 Una lectura naturalizada de la historia universal de Kant fue preconizada por el estudio de Paul Menzer, a quien le siguió Friedrick Kaulbach. Véase: MENZER, P. Kants Lehre von der Entwicklung in Natur und Geschichte. Berlín: Georg Reimer, 1911, p. 268; KAULBACH, F. Der Zusammenhang zwischen Naturphilosophie und Geschichtsphilosophie bei Kant. Kantstudien, 56, 1966, pp. 430, 434. Con el propósito de explicar los prejuicios contra la "naturalización" de la concepción de la historia humana, V. Gerhardt señala que "la reserva comprensible y muchas veces justificada contra el biologicismo hicieron difícil, luego de 1945, referirse al fundamento vital (das Lebensfundament) de la existencia humana". GERHARDT, V. Immanuel Kant. Vernunft und Leben. Stuttgart: Reclam, 2002, p. 245. Las itálicas son del autor. En la actualidad, defienden esta posición: WOOD, A. Kants Ethical Thought. USA: Cambridge University Press, 1999, p. 208; Unsociable Sociability: The Anthropological Basis of Kantian Ethics. Philosophical Topics. 19:1, 1999, p. 113; KLEINGELD, P. Fortschritt und Vernunft: Zur Geschichtsphilosophie Kants. Würzburg: Königshausen \& Neumann, 1995, pp. 126ss, 194, 214; COHEN, A. Kant and the Human Sciences. Biology, Anthropology and History. Hampshire: Macmillan, 2009, pp. XII, 119, etc. Se expresan, sin embargo, manifiestamente en contra de esta lectura: LANDGREBE, L. Die Geschichte im Denken Kants. Studium Generale, 7, 1954, p. 535; ANDERSON-GOLD, Sh. Teleology and Radical Evil: An Interpretation of the Concept of Species Character in Kant's philosophy of History. Michigan: University Microfilms International, 1981, pp. 3-5, 53; YOVEL, Y. Kant and the Philosophy of History, Princeton: Princeton University Press, 1989, p. 138; HONNETH, A. Kein Ende der Geschichte? Geschichtsphilosophie nach Kant. En KAPLOW, I. (ed.). Nach Kant: Erbe und Kritik. Münster: Lit Verlag, 2005, p. 128, entre otros. 
revisión de nuestra tesis según la cual la epigénesis es el modelo mediante el cual Kant explica el desarrollo de la historia universal o la cultura humana. ${ }^{4}$

\section{La epigénesis kantiana}

Aunque la "epigénesis" designa a una de las escuelas más importantes de historia natural del siglo XVIII es difícil reconstruir qué entendieron exactamente los hombres de ese tiempo por dicha posición. En líneas generales, sin embargo, los intérpretes acuerdan en que la epigénesis es una teoría que afirma una potencia originaria, una capacidad inmanente de la naturaleza de transformarse a sí misma. ${ }^{5}$ Para esta concepción la explicación de la producción de las variaciones orgánicas no requiere suponer, en contraposición a la escuela del preformismo, ciertas semillas y disposiciones que contengan en potencia la forma individual de la variación orgánica.

Hay evidencia de que Kant estaba familiarizado con la teoría de la epigénesis desde, por lo menos, la década de 1770 a partir de sus reflexiones sueltas. ${ }^{6}$ Ahora, la primera vez que Kant usa el término “epigénesis" es en la (primera) Recensión al primer volumen de Ideas para una filosofía de la historia de la humanidad (17845) de Herder. Aquí Kant usa el término en el marco de una cita textual de la obra de Herder sin indicar, sin embargo, cuál es su posición al respecto. ${ }^{7}$ Por eso, los intérpretes que buscan clarificar el concepto han recurrido a la Crítica de la facultad de juzgar (CFJ) en donde Kant menciona y ofrece una breve explicación de qué entiende por epigénesis.

En el contexto de discutir teorías alternativas del origen de las variaciones orgánicas en la historia natural Kant se alinea en el interior de la epigénesis que adjudica aquí al naturalista Blumenbach. ${ }^{8}$ Blumenbach defiende una concepción de

4 Con la excepción de la Crítica de la razón pura (CRP), se citan los textos de Kant siguiendo la siguiente edición la siguiente edición: Kants gesammelte Werke. Berlín: Preussische/Deutsche Akademie der Wissenschaften, 1902 ss. (la numeración romana indica el tomo y la arábiga, a la página). La CRP se cita, según el modo habitual: CRP A (edición 1781) y/o B (edición 1787), el número (romano o arábigo) se corresponde con la página.

5 ROE, SH. Matter, Life and Generation. Eighteenth-century embryology and the Haller-Wolff debate. Cambridge: Cambridge University Press, 1981, pp. 1-9. Véase allí sus referencias.

6 En las reflexiones de esta época se encuentran esparcidas algunas ideas relativas a la epigénesis: Kants gesammelte Werke, XVII, 416, 492, 554, 672; XVIII, 8, 12, 189, 273, 273-5, 423.

7 Kants gesammelte Werke, VIII, 050. La importancia del concepto de "epigénesis" se evidencia si atendemos a la sección "Deducción trascendental de los conceptos puros del entendimiento" de 1787 donde se presenta la manera como entiende la filosofía crítica la producción de las categorías como la "epigénesis de la razón pura". Véase: CRP, B 167. Un artículo reciente sobre el tema en donde se recoge abundante literatura sobre la discusión es: Lerussi, N. "Acerca de la analogía de la razón con lo orgánico. Reflexiones en torno a la expresión 'epigénesis de la razón pura' en Kritik der reinen Vernunft § 27”. En: CAIMI, M. (ed.). Estudios kantianos. Buenos Aires: Prometeo, 2014, pp. 113-136.

8 Blumenbach le envía a Kant en 1789, precisamente durante la redacción de la segunda parte de la CFJ, una copia de su libro Sobre el impulso formativo que el filósofo le agradece un año después en la carta fechada el 5.08.1790. Véase: Kants gesammelte Werke, XI, 184ss. 
esta escuela que entiende la formación orgánica a partir de la sola acción de un impulso formativo (Bildungstrieb) que se reproduce a sí mismo en función de las condiciones requeridas de tiempo y lugar, negando, así, la necesidad de suponer semillas y disposiciones preformadas en el organismo. ${ }^{9}$

Pues bien, aunque Kant no indique una corrección explícita a Blumenbach, el filósofo conserva, en la descripción de su versión de epigénesis, el concepto de ciertas "disposiciones". ${ }^{10}$ Por eso, la posición de Kant debe diferir de la concepción de Blumenbach: ${ }^{11}$ de hecho, Kant también denomina a su versión de epigénesis "preformismo genérico". Si bien es imprescindible, en relación a la comprensión de las variaciones orgánicas a lo largo del tiempo, asumir la acción de una fuerza formadora (o "impulso formativo" en la terminología de Blumenbach), irreductible al mecanismo, la cual se reproduce a sí misma en función de las circunstancias externas, esto no puede ser suficiente para completar la comprensión del modo como se produce, según la experiencia, la variación orgánica. Para esto, es necesario suponer, además, la acción de ciertas disposiciones.

De hecho, hay un pasaje importante en el parágrafo $\$ 80$ de la CFJ en donde Kant explica, al pasar, la formación orgánica de una modificación que expresa, a nuestro entender, los elementos contemplados en su versión de epigénesis en tanto preformismo genérico:

Aun en lo que atañe a la modificación a que son sometidos casualmente ciertos individuos de las especies organizadas, si se encuentra que su carácter, así modificado es hereditario y está incorporado en la fuerza reproductora (Zeugungskraft), aquello no puede ser juzgado de otra manera sino como desarrollo ocasional de una disposición conforme a fin originariamente presente en la especie para su auto-conservación. ${ }^{12}$

Las circunstancias externas dan cuenta de la dirección hacia la cual se modifican los individuos a ellas sometidos, sin embargo, las mismas no bastan para explicar por qué los individuos que han sido alterados pueden reproducir la modificación cuando las circunstancias externas posteriores son otras. Esta capacidad de

9 BLUMENBACH, F. Über den Bildungstrieb und das Zeugunskräfte. Göttingen: Johann Chr. Dietrerich, 1791 , pp. 14, 87, 98ss.

10 Sobre la discusión entre los críticos entre la relación entre Kant y Blumenbach, véase: RICHARDS, R. Kant and Blumenbach on the Bildungstrieb: A historical Misunderstanding. Studies in the History and Philosophy of Biology and Biomedical Sciences, 31, 2000, pp. 11-32; BERNASCONI, R. Kant and Blumenbach's Polyps. En: EIGEN, S.; LARRIMORE, M. (ed.). The German Invention of Race. Albany: State University of New York Press, 2006, p. 74; LENOIR, T. Kant, Blumenbach, and Vital Materialism in German Biology. Isis, vol. 71, Nro. 1, 1980, pp. 77-108, etc.

11 Kants gesammelte Werke, V, 423. Axin Cohen considera "que la posición de Kant se clasifica mejor como un punto intermedio entre el preformismo y el epigenetismo" pues la misma supone "ambos elementos epigenetista y preformistas". En este sentido, la posición de Kant sería "única". COHEN, A. Kant and the Human Sciences, p. 22.

12 Kants gesammelte Werke, V, 420. 
reproducir la alteración, sin la intervención de los factores externos implicados, debe corresponderse con una fuerza reproductora singular irreductible al mecanismo natural. Ahora bien, estos dos elementos por sí solos no alcanzan todavía para dar cuenta del modo como se producen las modificaciones según la experiencia. Esto es, específicamente, por qué la fuerza reproductora o formadora de una criatura que ha sido alterada por las condiciones externas reproduce necesariamente la variación, por qué una vez ocurrida una alteración específica no es posible dar marchar atrás. Como se deja traslucir, fundamentalmente, en los textos en los que el filósofo se ocupa de la cuestión de la producción orgánica de las variaciones de la especie humana como género natural, ${ }^{13}$ el desarrollo de especificaciones en el interior de una estirpe originaria es irreversible, esto es, los factores físicos externos no pueden, posteriormente, mutar los individuos hacia nuevas formas. Si la fuerza formadora no tuviera límites para modificarse, ocasionalmente, en función de las circunstancias, es decir, si pudieran estos dos factores, por sí solos, explicar la producción orgánica de las variaciones, como defiende Blumenbach, entonces, deberían los individuos poder alterarse hacia una nueva forma, indefinidamente, cada vez que se trasladaran a una nueva geografía. Pero esto es refutado por la experiencia. Los individuos que se han trasladado de geografía no pueden modificar reversiblemente su forma porque, tan pronto se han despertado ciertas disposiciones originarias, a través del condicionamiento geográfico de origen, la reproducción de la variación es necesaria. A partir de entonces, la modificación es dada en herencia, para siempre, en sus descendientes futuros. Pero esto significa que ciertas disposiciones deben ser supuestas en toda teoría epigenética que pretenda dar cuenta de la experiencia. De esta manera, la concepción kantiana de epigénesis que se deja entrever en la CFJ contiene los conceptos de una fuerza vital interna de reproducción, originariamente productora de sí misma, las circunstancias externas que pueden desviar la reproducción hacia una variación y ciertas disposiciones originales que definen las limitaciones dentro de las cuales las modificaciones pueden producirse.

Ahora bien, a diferencia de la concepción que de las disposiciones y semillas tiene el preformismo (lo que Kant llama en \$81 de la CFJ, "preformismo individual" o "teoría de la evolución"), según la cual aquellas son como capullos que contienen de manera íntegra y acabada, aunque en potencia, la forma del individuo, según la concepción kantiana, las disposiciones no son más que “limitaciones” que restringen la fuerza interna de reproducción de variaciones en una criatura. Ellas no contienen en potencia la forma completa de los individuos, sino sólo la forma según el género, esto es, definen el radio en el interior del cual el individuo puede variar y, al mismo tiempo, el marco fuera del cual las modificaciones ya no son posibles. En otros

13 Se trata de los textos de Kant concernientes a las "raza humana", véase: Kants gesammelte Werke, II, 441; VIII, 098. 
términos, delimitan la estructura dentro de la cual los seres organizados se organizan. Se trata, consecuentemente, de una función meramente restrictiva que acompaña la función espontánea y creativa de la fuerza o impulso interno de producción de sí de los seres orgánicos.

\section{Disposiciones naturales de la especie humana y circunstancias de desarrollo según Idea de una historia universal en sentido cosmopolita (Idea)}

En Idea el filósofo propone introducir una reflexión acerca de la marcha de la especie humana bajo el presupuesto de un plan o propósito de la naturaleza. El punto central de la argumentación es mostrar que dicho plan o propósito asignado a la naturaleza no actúa en el vacío como un deus ex machina presionando desde afuera el curso de las acciones humanas sino que se inscribe en ciertas "disposiciones originales" de la especie. De allí que Kant inaugure la problemática de la historia universal mediante los conceptos de "disposiciones originales" o "disposiciones naturales”, que serán centrales entre las tesis primera y cuarta. Así, en la primera tesis el filósofo afirma que todas las disposiciones de un ser orgánico han de desarrollarse en algún momento de manera completa y conveniente. ${ }^{14}$ En la segunda tesis añade, sin embargo, que puesto que las disposiciones destinadas al uso de la razón en el hombre se desarrollan de forma ilimitada y que los seres humanos son mortales, las primeras no se desarrollan de forma completa individualmente, sino sólo en la especie. Estas dos tesis son las premisas de la afirmación hacia el final de la tesis tercera cuando asegura que es necesaria la idea de que las generaciones pasadas de la humanidad deban laborar para la fortuna de las generaciones futuras.

Pues bien, en la tesis cuarta de Idee Kant se ocupa de comprender el tránsito desde el estado de rudeza de la especie hacia el estado de la cultura ${ }^{15}$ a partir del concepto de "disposición" inscripta en la "naturaleza humana". Las disposiciones juegan aquí un doble papel pues no sólo son aquello que se debe desarrollar en el tiempo, sino también el medio o la circunstancia mediante el cual las mismas se desarrollan. Así, el paso de la animalidad hacia la humanidad del hombre es comprendido mediante el "antagonismo de las disposiciones en sociedad" del que finalmente resulta un ordenamiento legal que es condición, a la vez, para el desarrollo de "todas las disposiciones" 16 de la especie. Veamos:

El medio del que se sirve la naturaleza para conducir al desarrollo de todas sus disposiciones es el antagonismo de las mismas en sociedad hasta tal punto que será finalmente la causa de un orden conforme a ley de la misma. ${ }^{17}$

14 Kants gesammelte Werke, VIII, 018.

15 Una definición amplia de "cultura" se encuentran en: Kants gesammelte Werke, V, 430, 431.

16 Kants gesammelte Werke, VIII, 022.

17 Kants gesammelte Werke, VIII, 020. 
Kant señala inmediatamente a continuación que el "antagonismo de las disposiciones en sociedad" es equivalente al concepto de "insociable sociabilidad" (ungesellige Geselligkeit) que, según defiende aquí Kant, es un conjunto de disposiciones que se reducen a la "propensión a ingresar a la sociedad unida a una resistencia continua" 18 a entrar a la misma. La "insociable sociabilidad" está compuesta, así, por un lado, por una disposición social del hombre a reunirse en sociedad, esto es, una inclinación a estar en compañía de los otros y, por otro lado, por la disposición insociable que consiste en una inclinación a aislarse y a vivir según el propio sentido. Kant señala:

El hombre tiene una propensión a socializarse; porque en este estado siente más su condición de hombre; es decir, tiene el sentimiento de desarrollar sus disposiciones naturales. Pero también posee una gran inclinación a individualizarse (aislarse), porque, al mismo tiempo, encuentra en él una cualidad insociable de querer dirigir todo simplemente según su modo de pensar. ${ }^{19}$

Según se expresa en este último texto las disposiciones a la sociabilidad y a la insociabilidad son, en primer lugar, primarias o irreductibles una a otra, son, de hecho, disposiciones opuestas. En segundo lugar, no se le otorga a ninguna de ellas una connotación moral. Las disposiciones sociables e insociables refieren, respectivamente, a una inclinación o a un rechazo hacia la compañía de los otros y no a un deseo de hacerles el bien o hacerles daño, de competir o cooperar con ellos.

Ahora bien, la disposición a aislarse degenera tan pronto se pone en tensión con la disposición sociable, pues seres que tienen la inclinación a vivir según el propio modo de pensar querrán continuar haciéndolo cuando se encuentren, por el principio de la sociabilidad, en comunidad con los otros. Por eso, se dice en Idea que el hombre en sociedad se encuentra en un permanente estado de alerta, ya que “espera resistencia por todos lados, puesto que sabe por sí mismo que él, en lo que le incumbe, está inclinado a resistirse a los demás". ${ }^{20}$ El hombre en sociedad se resiste frente a los demás porque sabe que los demás querrán, como él, vivir según su propio criterio en sociedad. Más aún, es la presunción de que los otros pretendan imponerle su propio sentido o modo de pensar lo que inclina al hombre a entrar en una carrera por el rango entre sus congéneres, a un estado de competencia por obtener los medios que otorgan superioridad al hombre y que facilitan la imposición del propio sentido. Es a partir de esta competencia por el rango de donde surge una tríada de pasiones que implican las tres formas centrales de competencia entre los hombres,

18 Kants gesammelte Werke, VIII, 020.

19 Kants gesammelte Werke, VIII, 020/1.

20 Kants gesammelte Werke, VIII, 021. 
esto es, la competencia por la riqueza, por el dominio y por el honor. ${ }^{21}$ Así, mediante la "ambición, el afán de dominio o la codicia” se produce, finalmente, “los primeros pasos hacia la cultura"22. Así:

Tal resistencia despierta todas las fuerzas del hombre y lo lleva a superar la inclinación a la pereza. Impulsado por la ambición, el afán de dominio o la codicia, llega a procurarse cierta posición (Rang) entre sus asociados a los que, en verdad, no puede soportar, pero tampoco evitar. De esta manera se dan los primeros pasos verdaderos que llevan de la grosería a la cultura. ${ }^{23}$

Aunque por el principio a la sociabilidad, el hombre quiere vivir sosegado y divertido, en una "inercia e inactiva satisfacción", por su disposición a la insociabilidad en el contexto de la sociedad debe salir de la indolencia y del inactivo contento, a fin de que "que se entregue al trabajo y los penosos esfuerzos para encontrar los medios, como desquite, de librarse sagazmente de tal condición". ${ }^{24} \mathrm{De}$ este modo, mediante los frutos del trabajo fatigoso, riquezas, dominio y honores, el hombre puede encontrar una ventaja en relación a sus congéneres perezosos $\mathrm{y}$, por consiguiente, facilitar la imposición del propio sentido o modo de pensar.

De lo dicho se sigue que si los hombres tuvieran exclusivamente una disposición a la sociabilidad vivirían pacíficamente en un estado de pereza generalizada, en una "arcádica vida de pastores". Si los hombres tuvieran sólo una disposición insociable se dispersarían a través del globo sin fundar comunidad alguna, se aislarían. Por eso, la tensión entre la disposición sociable y la disposición insociable o la degeneración de la disposición insociable, en el contexto de la sociedad, es aquello que permite explicar el ingreso del hombre a la cultura. ${ }^{25}$

21 La competencia por el rango o por la posición como fuente de la cultura ha sido inscripta, por algunos autores, en el marco de una tradición, que va de Rousseau a Hegel, según la cual lo humano se define a partir de una "lucha por el reconocimiento". Véase: YOVEL, Y. Kant and the Philosophy of History, p. 148ss; HONNETH, A. „Kein Ende der Geschichte?“, p. 129. Esta visión encuentra una fuente importante, a nuestro entender, en la Antroplogía en sentido pragmática (Antroplogía) cuando el filósofo entiende que las tres pasiones nombradas son "inclinaciones de la ilusión" que "consiste en equiparar la mera opinión ajena sobre el valor de las cosas [léase: el honor, el dominio y la riqueza] a su verdadero valor". Kants gesammelte Werke, VII, 270. Los corchetes son añadido nuestro. De esta manera, la competencia por obenter los medios que otorgan superioridad (rango) a los hombres es una competencia, en realidad, por obtener los medios que, en la opinión de los hombres, otorgan dicha superioridad. Se trata así de una competencia por las opiniones.

22 Kants gesammelte Werke, VIII, 021.

23 Kants gesammelte Werke, VIII, 021.

24 Kants gesammelte Werke, VIII, 021.

25 En esta misma tesis cuarta Kant dice más abajo, en una dirección aparentemente contraria a la que estamos defendiendo, que "sin la mencionada cualidad de la insociabilidad (...) por la que surge la resistencia (...), en virtud de pretensiones egoístas, todos los talentos hubiesen quedado ocultos por la eternidad”. Kants gesammelte Werke, VIII, 021. Véase además: Kants gesammelte Werke, VIII, 022, 024. Sin embargo, repetimos, al costo de ser reiterativos, que es la insociable sociabilidad y no la insociabilidad sola aquello que se encuentra en la base de la competencia por el rango de la cual surge la cultura. La insociabilidad sola nos vuelve ermitaños, no competitivos. Nuestra 
Pues bien, en este esquema vemos que los conceptos centrales de comprensión de la epigénesis juegan un papel fundamental también en la consideración de las variaciones de la especia humana como género racional. Las disposiciones naturales y las circunstancias de desarrollo de las disposiciones son centrales en el modelo que el filósofo utiliza para dar cuenta del devenir histórico en Idea. No obstante esto, hay una diferencia que es importante subrayar en la concepción que el filósofo tiene aquí de las circunstancias promotoras en comparación con la concepción que de ellas tiene en su historia natural. En la historia universal las circunstancias externas son concebidas como producto del género humano, esto es, como una consecuencia del mismo desarrollo de las disposiciones naturales de la especie y no, como en la historia natural, como factores físicos externos que los miembros de la especie se encuentran diferencial y casualmente. Como hemos señalado ya, en el título de la tesis cuarta de Idea, Kant deja constancia de que las disposiciones naturales de la especie humana como género racional no sólo son aquello que se desarrolla en la historia sino también son el medio a través del cual las mismas se desarrollan. Es, precisamente, este factor autorreferencial de las disposiciones para el uso de la razón lo que explica por qué en la sección tercera de Idea se insiste en que la naturaleza ha querido respecto del hombre que él extraiga de sí mismo todo lo que sobrepasa su existencia animal "y que no participe de ninguna otra felicidad o completitud que aquella que él mismo, libre del instinto, pueda proveerse mediante la razón". ${ }^{26} \mathrm{El}$ hombre como ser racional es un producto de sí mismo en un sentido más restringido que el hombre como especie meramente natural que es un producto conjunto de sí mismo y de los factores naturales externos que casualmente encuentra. Esto es así porque las circunstancias externas son producto del desarrollo mismo de la especie: el desarrollo de ciertas disposiciones produce un estado de cosas (A) que Kant denomina “tensiones de fuerzas” (Anspannung der Kräfte) ${ }^{27}$ - que despierta disposiciones aún dormidas cuyo desenvolvimiento dará en un nuevo estado de cosas (B) - “nuevas tensiones de fuerza”- que será punto de partida para el desarrollo de

explicación del concepto de "insociable sociabilidad", difiere, sin embargo, de la ofrecida por otros intérpretes. Véase: WOOD, A. Unsociable Sociability: The Anthropological Basis of Kantian Ethics; Kant's Fourth Proposition: the unsociable sociability of human nature. En: RORTY, A. O.; SCHMIDT, J. (ed.). Kant's Idea for the Universal History with a Cosmopolitan Aim. A Critical Guide. New York: Cambridge University Press, 2009; PHILONENKO, A. La théorie kantienne de l'histoire. Paris: Vrin, 1986; KLEINGELD, P. Fortschritt und Vernunft; Belwe, A. Ungesellige Geselligkeit. Kant: Warum die Menschen einander „nicht wohl leiden“, aber auch „nicht voneinander lassen“ können. Würzburg: Königshausen \& Neumann, 2000; LOUDEN, R. Kant's impure Ethics: From rational Beings to human Beings, New York/Oxford: Oxford University Press, 2000; YOVEL, Y. Kant and the Philosophy of History, etc. En nuestro texto, aún inédito, "Insociable sociabilidad y cultura humana en la tesis IV de Idea de una historia universal en sentido cosmopolita de I. Kant" hemos discutido estas interpretaciones alternativas y mostrado por qué nuestra interpretación se ajusta mejor a la letra kantiana.

26 Kants gesammelte Werke, VIII, 019.

27 Kants gesammelte Werke, VIII, 019. 
nuevas disposiciones. Consecuentemente, se inscriben en la especie humana como género racional dispositivos de autoproducción de sí en un sentido enfático. ${ }^{28}$

Entre la tesis cuarta y octava de Idea se destacan una serie de factores externos, "relaciones de fuerza" o circunstancias culturales mediante las cuales son promovidas diferencialmente las disposiciones naturales de los hombres. Significativamente, en la sección cuarta, quinta y octava de Idee, Kant presenta lo que podría llamarse un tipo progresista de historia según el cual el paso de cada circunstancia cultural a la siguiente, esto es, de la rudeza a un orden legal, de éste, a una sociedad civil plenamente justa $y$, finalmente, de ésta, a una sociedad moral en donde pueden desarrollarse todas las disposiciones humanas, se produce a partir de las condiciones generadas por las circunstancias culturales previas.

En la tesis cuarta de Idea Kant indica abruptamente que el efecto que tendrían ciertos productos de la cultura, e.d. del arte y la ciencia, es producir un cierto sentimiento (la formación de gusto) y un nuevo modo de pensar, en general. ${ }^{29}$ Una sociedad patológicamente formada en la que los hombres compiten por producir productos culturales, a la larga, a través de la apreciación y discusión pública de sus productos, hace nacer una nueva afectividad y modo de pensar que "puede transmutar con el tiempo las disposiciones naturales rudas hacia la discriminación moral", que es base de una "sociedad en un todo moral". ${ }^{30}$ De este modo, una sociedad del gusto y de la ilustración, resultante del estado de competencia tendría por consecuencia, la moralidad y el consecuente desarrollo de todas las disposiciones humanas. ${ }^{31}$ ¿Cuáles son los pasos intermedios entre la salida del hombre del estado de rudeza y el ingreso a una sociedad moral en la que pueda desarrollar el hombre todos sus talentos?

Es una condición previa para el ingreso a una sociedad moral la institución de una "constitución civil plenamente justa". En la tesis octava Kant ofrece dos líneas de argumentos por las cuales, en los Estados patológicamente formados por la naturaleza competitiva del hombre, se desenvuelven dispositivos no morales (naturales, entonces) que generan, finalmente, las condiciones para instituir una constitución civil plenamente justa. Según la primera línea argumental, Kant muestra cómo, desde la libertad civil de comerciar surgiría un estado de libertad más general, religiosa y de pensamiento -la ilustración- del que resultaría "un sentimiento en todos" los miembros del Estado, incluso en sus gobernantes, por "la conservación del conjunto". Específicamente, este sentimiento consistiría en

28 Sobre la auto-producción de la especie humana, véase: GERHARDT, V. Immanuel Kant, pp. 325/6; WOOD, A. Kant's Fourth Proposition: the unsociable sociability of human nature, p. 114. Ahora, la autoproducción de sí del hombre no es una invención de la naturaleza del hombre sino producción de la especificidad del hombre que es un desarrollo diferencial de las disposiciones dadas al género por la naturaleza.

29 Kants gesammelte Werke, VIII, 021.

30 Kants gesammelte Werke, VIII, 021.

31 Esta tesis se repite en: Kants gesammelte Werke, V, 433ss. 
una "cierta participación cordial en lo bueno que debe ascender hasta trono y tener una influencia (...) en los principios de gobierno". ${ }^{32}$ Así, todos los miembros del Estado, incluso sus gobernantes, tendrían una motivación sentimental por instituir una constitución civil plenamente justa. La segunda línea argumental muestra cómo la situación de los Estados en guerra o en peligro de guerra se volverá cada vez más inconveniente. El peligro financiero e industrial generado por la guerra o su peligro conduciría, entonces, a los Estados patológicamente formados hacia un "futuro gran cuerpo de Estado", hacia una "situación general cosmopolita": 33 se trata de un estado internacional de "tranquilidad y seguridad", de "seguridad y derecho". ${ }^{34}$ Mediante esta sintética argumentación Kant muestra cómo a partir de la competencia entre los hombres, producto de su insociable sociabilidad, pueden ellos alcanzar la institución de constituciones civiles plenamente justas, tanto en lo interno como en las relaciones externas, base de una sociedad moral en donde, finalmente, puedan desarrollarse plenamente todas disposiciones humanas, incluso las disposiciones para la moralidad.

\section{Epigénesis e historia universal}

En lo precedente hemos mostrado que tanto en la explicación de las variaciones de la especie humana como género natural como en la comprensión de las variaciones (culturales) de la especie humana como género racional, Kant utiliza la misma matriz de comprensión. Notemos, sin embargo, que en la comprensión de la historia universal el filósofo hace uso de sólo dos de los conceptos incluidos en la teoría de la epigénesis, es decir, las “disposiciones naturales u originales" y las "circunstancias externas". Esto no implica suponer que Kant utiliza aquí un modelo epigenético más reducido, que excluye el concepto de una “fuerza formadora”. De hecho, las disposiciones naturales son estructuras de variaciones de un ser capaz de incorporar, reproducir y producir, dichas variaciones a su propia fuerza de acción. Que este es el caso también en la historia universal se evidencia si consideramos cómo entiende Kant el proceso de "ininterrumpida transmisión" cultural entre las generaciones. Cada generación no comienza desde cero su propia historia, por el contrario, cada generación incorpora las condiciones culturales heredadas por las generaciones pasadas y las reproduce con variaciones cuando las entrega en legado a las generaciones futuras. Por eso, la posibilidad de desarrollar plenamente las disposiciones para el uso de la razón implica la capacidad de incorporar las formas culturales previas y modificarlas, esto

32 Kants gesammelte Werke, VIII, 028.

33 Kants gesammelte Werke, VIII, 024/028. Sobre el“espíritu comercial” y el "poder del dinero", véase: Kants gesammelte Werke, V, 433; VIII, 368.

34 Sobre la posición kantiana acerca de la utilidad de la guerra, véase: Kants gesammelte Werke, VIII, 120ss, 310ss., 363ss., 367; V, 432ss. 
es, de reproducirlas y de producir una diferenciación de ellas y, por lo tanto también, supone el concepto de una fuerza formadora.

Así, en lo precedente hemos mostrado que la matriz conceptual utilizada en la concepción de la historia universal en el texto Idea es la misma mediante la cual el filósofo da cuenta del desarrollo de los seres organizados y de la especie humana como género natural según la teoría de la epigénesis. Hablamos así de una concepción naturalizada de la filosofía de la historia de Kant. Esto no implica, sin embargo, una reducción de la cultura al reino de una naturaleza externa y enajenadora. Por el contrario, nos enfrentamos a una concepción de la naturaleza humana como fuerza racional productiva e interna, capaz de darse su propia forma, de formarse a sí misma. Según esta concepción se inscribiría en la naturaleza humana como género racional una fuerza singular, estructurada por disposiciones naturales hacia el uso de la razón de cuya tensión se producirían diferentes relaciones de fuerzas o circunstancias culturales externas que permitirían, tras un largo camino, la institución de una sociedad civil plenamente justa y, por ella mediante, la formación de una sociedad moral en la que todas las disposiciones de los hombres puedan desarrollarse.

\section{Referencias}

KANT, I. Kants gesammelte Werke. Berlín: Preussische/Deutsche Akademie der Wissenschaften, 1902ss.

\section{Bibliografía secundaria}

ANDERSON-GOLD, Sh. Teleology and Radical Evil: An Interpretation of the Concept of Species Character in Kant's philosophy of History. Michigan: University Microfilms International, 1981.

BELWE, A. Ungesellige Geselligkeit. Kant: Warum die Menschen einander „nicht wohl leiden“, aber auch „nicht voneinander lassen“ können. Würzburg: Königshausen \& Neumann, 2000.

BERNASCONI, R. Kant and Blumenbach's Polyps. En EIGEN, S.; LARRIMORE, M. (ed.). The German Invention of Race, Albany: State University of New York Press, 2006.

BLUMENBACH, Jh. F. Über den Bildungstrieb und die Zeugungskräfte. Göttingen: Johann Chr. Dietrerich, 1791.

COHEN, A. Kant and the Human Sciences. Biology, Anthropology and History. Hampshire: Macmillan, 2009.

HONNETH, A. Kein Ende der Geschichte? Geschichtsphilosophie nach Kant. En: KAPLOW, I. (ed.). Nach Kant: Erbe und Kritik. Münster: Lit Verlag, 2005.

KAULBACH, F. DerZusammenhangzwischenNaturphilosophieundGeschichtsphilosophie 
bei Kant. Kantstudien, 56, 1966.

KLEINGELD, P. Fortschritt und Vernunft: Zur Geschichtsphilosophie Kants. Würzburg: Königshausen \& Neumann, 1995.

GERHARDT, V. Kulturelle Evolution. Philosophische Anmerkungen zu einem nicht erst seit Darwin aktuellen Programm. En GEHARDT, V.; NIDA-RUMELIN (ed.). Evolution in Natur und Kultur. Berlin: De Gruyter, 2010. Immanuel Kant. Vernunft und Leben. Stuttgart: Reclam, 2002.

LANDGREBE, L. Die Geschichte im Denken Kants. Studium Generale, 7, 1954.

LENOIR, T. Kant, Blumenbach, and Vital Materialism in German Biology. Isis, 71, 1, 1980.

LERUSSI, N. Acerca de la analogía de la razón con lo orgánico. Reflexiones en torno a la expresión 'epigénesis de la razón pura' en Kritik der reinen Vernunft $\S 27$. En: CAIMI, M. (ed.). Estudios kantianos. Buenos Aires: Prometeo, 2014, pp. 113-136.

LOUDEN, R. Kant's impure Ethics: From rational Beings to human Beings. New York/ Oxford: Oxford University Press, 2000.

MENZER, P. Kants Lehre von der Entwicklung in Natur und Geschichte. Berlín: Georg Reimer, 1911.

PHILONENKO, A. La théorie kantienne de l'histoire. Paris: Vrin, 1986.

RICHARDS, R. Kant and Blumenbach on the Bildungstrieb: A historical Misunderstanding. Studies in the History and Philosophy of Biology and Biomedical Sciences, 31, 2000.

ROE, SH. Matter, Life and Generation. Eighteenth-century embryology and the Haller-Wolff debate. Cambridge: Cambridge University Press, 1981.

WIEGEL, S. An der Schwelle von Kultur und Natur. Epigenetik und Evolutionstheorie. En GEHARDT, V.; NIDA-RUMELIN (ed.). Evolution in Natur und Kultur. Berlin: De Gruyter, 2010.

WOOD, A. Kants Ethical Thought. USA: Cambridge University Press, 1999.

Unsociable Sociability: The Anthropological Basis of Kantian Ethics. Philosophical Topics, 19:1, 1999.

Kant's Fourth Proposition: the unsociable sociability of human nature. En: RORTY, A. O.; SCHMIDT, J. (ed.). Kant's Idea for the Universal History with a Cosmopolitan Aim. A Critical Guide. New York: Cambridge University Press, 2009.

YOVEL, Y. Kant and the Philosophy of History, Princeton: Princeton University Press, 1989. 